\title{
Bending Deflection Analysis of a Semi-Trailer Chassis by Using Symmetric Smoothed Particle Hydrodynamics
}

\author{
Armagan Karamanli** \\ *Research and Development Department, TIRSAN Treyler Sanayi ve Ticaret A.S., Sancaktepe, Istanbul, Turkey \\ armagan_k@yahoo.com
}

\author{
${ }^{\ddagger}$ Corresponding Author; Armagan Karamanli, Osmangazi Mah., Yıldızhan Cad., No:4, 3488, Sancaktepe, Istanbul, Turkey \\ Tel: +90 216564 0200, Fax: +90 216311 7156, armagan_k@yahoo.com
}

Received: 05.10.2015 Accepted: 08.11.2015

\begin{abstract}
In this paper, a simple approach is presented for the calculation of bending deflection of a semi trailer chassis. The 3D model of the chassis is used to obtain the function of the moment of inertia and then the mathematical model of the chassis is presented as an Euler Bernoulli Beam which has the variable cross section. Different loading conditions raised from the semi trailer test procedures are applied. The bending deflections of the semi trailer chassis are numerically calculated by using the Symmetric Smoothed Particle Hydrodynamics (SSPH) method. The first time, the performance of the SSPH method for the fourth order non-homogeneous variable coefficents linear boundary value problems is evaluated. For the calculations different numbers of terms in the TSEs are employed when the number of nodes in the problem domain increases. The comparisons are made with the results of experiments. It is observed that the SSPH method has the conventional convergence properties and yields smaller $\mathrm{L}_{2}$ error. Finally, the approach presented here may be used for the calculation of deflection of the semi trailer chassis before the release of detail design.
\end{abstract}

Keywords Meshless methods, strong form, Taylor series expansion, element free method, computational mechanics.

\section{Introduction}

The choice of basis functions is one of the most important issues to obtain the approximate solution of an initial boundary value problem in numerical methods. One can improve the accuracy of the numerical solution either by increasing number of nodes or by increasing the degree of complete polynomials which are defined piecewise on the problem domain in the Finite Element Method. To find an approximate solution of an initial boundary value problem the basis functions to be used in meshless methods can be derived by Smoothed Particle Hydrodynamics (SPH) method, proposed by Lucy [1], Corrected Smoothed Particle Method (CSPM) [2, 3], Reproducing Kernel Particle Method (RKPM) [4-6], Modified Smoothed Particle Hydrodynamics (MSPH) method [7-10], the SSPH method [11-14] and the Strong Form Meshless Implementation of Taylor Series Method (SMITSM) [15-16], Moving Kringing Interpolation Method [17-18], the meshless Shepard and Least Squares (MSLS) Method [19].

The locations of nodes are only the parameters which are necessary to construct basis functions in the SSPH method. These basis functions can be found similar to those in the
Finite Element Methods however the derivatives of a function can be found without differentiating the basis function. Of course, the basis for the derivatives of a function can be obtained by differentiating the basis for the function as in the Finite Element Methods and meshless methods.

Because of the formulation of the Symmetric Smoothed Particle Hydrodynamics (SSPH) method the matrix to be inverted becomes symmetric and this reduces the CPU time. Moreover, the SSPH method eliminates the choice of weight function which must not be a constant. The SSPH method depends on the Taylor Series Expansion and calculates the value of the solution at a node by using the values of the solution at the other nodes and then substitutes it into the governing differential equation. The SSPH method has been successfully applied to 2D homogeneous elastic problems including quasi-static crack propagation [11-13] and 2D Heat Transfer problems.

A semi trailer chassis has a very complex structure and the structural analyses based on the bending deflections are generally performed by using commercial Finite Element Analysis software. This activity is costly and time consuming. Since the less deflection becomes a unique 
selling point of a semi trailer, during conceptual and detail design phases of the new product development process the mentioned analysis should be performed to obtain an acceptable chassis design which is validated by a series of tests. In this paper, an approach which is simple and requires less effort than the Finite Element Methods is presented for the calculation of deflection of a semi trailer chassis. First of all, by using the $3 \mathrm{D}$ data of the chassis a function for the moment of inertia of the cross section is created and then the chassis is modelled as Euler Bernoulli Beam. Different loading conditions which cause bending for the semi trailer chassis coming from the semi trailer test procedures are applied. The bending deflections of the semi trailer chassis are numerically calculated by using the SSPH method. Also, the performance of the SSPH method is evaluated by employing different number of terms in the associated Taylor Series Expansions and the calculation of deflection of a semi trailer chassis is studied then, comparisons are made with the results of experiments.

In section 2, the formulation of the SSPH method is presented for 1D application. In section 3 , the chassis of the semi trailer is modelled as a beam based on Euler Bernoulli beam theory. The moment of inertia of the Euler Bernoulli beam is defined as a function by using the moment of inertia values of totally 23 sections due to the non-uniform structure of the semi trailer chassis. In Section 4, two types of loading conditions are investigated. The performance of the Symmetric Smoothed Particle Hydrodynamics (SSPH) method is compared with the experimental results.

\section{Formulation}

If a function $\mathrm{f}(\mathrm{x})$ is continuous and differentiable up to the $(n+1)^{\text {th }}$ order, through the Taylor Series Expansion (TSE) the value of the function at a point $\xi=\left(\xi_{1}\right)$ located in the neighborhood of $\mathrm{x}=\left(x_{1}\right)$ can be approximated as following

$$
f\left(\xi_{1}\right)=\sum_{m=0}^{n} \frac{1}{m !}\left[\left(\xi_{1}-x_{1}\right) \frac{\partial}{\partial x_{1}}\right]^{m} f\left(x_{1}\right)
$$

If the eight and higher order terms are neglected, and matrices $\mathbf{P}(\xi, \mathrm{x})$ and $\mathbf{Q}(\mathrm{x})$ are introduced, one can write equation (1) as

$$
f(\xi)=\boldsymbol{P}(\xi, x) \boldsymbol{Q}(x)
$$

Where

$$
\begin{aligned}
\boldsymbol{Q}(x) & =\left[f(x), \frac{d f(x)}{d x_{1}}, \frac{1}{2 !} \frac{d^{2} f(x)}{d x_{1}^{2}}, \ldots, \frac{1}{n !} \frac{d^{7} f(x)}{d x_{1}^{7}}\right]^{T} \\
\boldsymbol{P}(\xi, x) & =\left[1,\left(\xi_{1}-x_{1}\right),\left(\xi_{1}-x_{1}\right)^{2}, \ldots,\left(\xi_{1}-x_{1}\right)^{7}\right]
\end{aligned}
$$

The unknown variables which are the elements of the $\mathbf{Q}(\mathrm{x})$, the estimate of the function, its first derivatives to seventh derivatives at $\mathrm{x}=\left(x_{1}\right)$ can be found from equation (2).

Both sides of equation (2) are multiplied with $W(\xi, \mathrm{x}) \mathbf{P}(\xi, \mathrm{x})^{T}$ and the following equation is obtained.

$$
\begin{array}{r}
f(\xi) W(\xi, x) \boldsymbol{P}(\xi, x)^{T}=\boldsymbol{P}(\xi, x) \boldsymbol{Q}(x) W(\xi, x) \boldsymbol{P}(\xi, x)^{T}, \\
=\left[\boldsymbol{P}(\xi, x)^{T} W(\xi, x) \boldsymbol{P}(\xi, x)\right] \boldsymbol{Q}(x)
\end{array}
$$

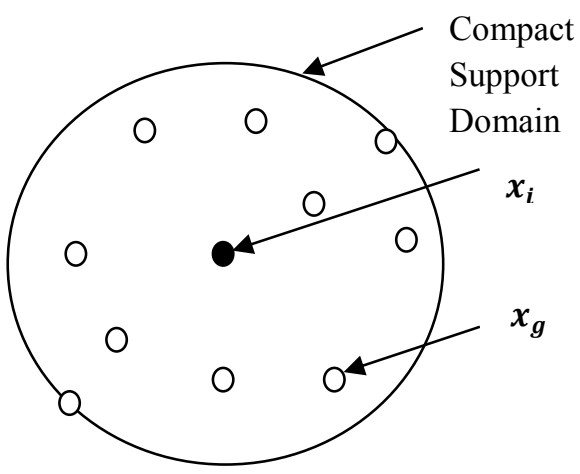

Fig. 1. Distribution of the nodes in the compact support of the kernel function $\mathrm{W}(\xi, \mathrm{x})$ associated with the point

$$
\mathrm{x}=\left(\mathrm{x}_{\mathrm{i}}, \mathrm{y}_{\mathrm{i}}\right)
$$

In the compact support domain of the weight function $W(\xi, \mathrm{x})$ associated with the point $\mathrm{x}=\left(x_{1}\right)$ shown in Figure 1 , let there be $N(\mathrm{x})$ nodes and $g(j)$ is the $j^{\text {th }}$ node in the compact support of $W(\xi, \mathrm{x})$. Equation (5) is evaluated at every node in the compact support domain of the $W(\xi, \mathrm{x})$. By summation of each side over these nodes to find out

$$
\begin{gathered}
\sum_{j=1}^{N(x)} f\left(\xi^{g(j)}\right) W\left(\xi^{g(j)}, \boldsymbol{x}\right) \boldsymbol{P}\left(\xi^{g(j)}, x\right)^{T} \\
=\sum_{j=1}^{N(x)}\left[\boldsymbol{P}\left(\xi^{g(j)}, x\right)^{T} W\left(\xi^{g(j)}, x\right) \boldsymbol{P}\left(\xi^{g(j)}, x\right)\right] \boldsymbol{Q}(x)
\end{gathered}
$$

Where $\xi^{g(j)}$ defines the coordinates of the node $g(j)$. By using the following definitions

$$
\begin{gathered}
\boldsymbol{H}(\xi, x)=\left[\boldsymbol{P}^{T}\left(\xi^{g(1)}, x\right), \boldsymbol{P}^{T}\left(\xi^{g(2)}, x\right), \ldots, \boldsymbol{P}^{T}\left(\xi^{g(N(x))}, x\right)\right], \\
\boldsymbol{W}(\xi, x)=\left[\begin{array}{ccc}
W\left(\xi^{g(1)}, x\right) & \cdots & 0 \\
\vdots & \ddots & \vdots \\
0 & \cdots & W\left(\xi^{g(N(x))}, x\right)
\end{array}\right], \\
\boldsymbol{F}^{(\boldsymbol{x}) T}(\xi, x)=\left[f\left(\xi^{g(1)}\right), f\left(\xi^{g(2)}\right), \ldots ., f\left(\xi^{g(N(x))}\right](7)\right.
\end{gathered}
$$

Equation (6) can be written as

$$
\begin{array}{r}
\boldsymbol{H}(\xi, x) \boldsymbol{W}(\xi, x) \boldsymbol{F}^{(x)}(\xi, x)= \\
\boldsymbol{H}(\xi, x) \boldsymbol{W}(\xi, x) \boldsymbol{H}(\xi, x)^{T} \boldsymbol{Q}(x)
\end{array}
$$

The values of the matrix $\mathbf{P}(\xi, \mathrm{x})$, the weight function $W(\xi, \mathrm{x})$ and the function $f$ at all nodes located in the compact support domain of $W(\xi, \mathrm{x})$ associated with point $\mathrm{x}$ are the elements which determine the values of element matrices $\mathbf{H}(\xi, \mathrm{x}), \mathbf{W}(\xi, \mathrm{x})$ and $\mathbf{F}^{(\boldsymbol{x})}(\xi, \mathrm{x})$. Then, equation (8) can be written as

$$
\boldsymbol{C}(\xi, x) \boldsymbol{Q}(x)=\boldsymbol{D}(\xi, x) \boldsymbol{F}^{(x)}(\xi, x)
$$

Where $\mathbf{C}(\xi, \mathrm{x})=\mathbf{H}(\xi, \mathrm{x}) \mathbf{W}(\xi, \mathrm{x}) \mathbf{H}(\xi, \mathrm{x})^{T} \quad$ and $\quad \mathbf{D}(\xi, \mathrm{x})=$ $\mathbf{H}(\xi, \mathrm{x}) \mathbf{W}(\xi, \mathrm{x})$.

It can be easily seen that the matrix $\mathbf{C}(\xi, \mathrm{x})$ defined above is symmetric. That's why this method is called as the SSPH method. The simultaneous linear algebraic equations given in equation (3.9) can be solved to obtain the unknown elements of the $\mathbf{Q}(\mathrm{x})$. The matrix $\mathbf{C}(\xi, \mathrm{x})$ to be inverted is symmetric. Because of symmetry property of the matrix $\mathbf{C}(\xi, \mathrm{x})$, the $\mathrm{CPU}$ 
time which is needed to solve equation (9) for the unknown elements of the $\mathbf{Q}(\mathrm{x})$ can be reduced. The matrices given in equation (9) do not include the derivatives of the weight function. By using a much larger class of weight functions including a constant the implementation and usefulness of the method can be improved.

For the non-singular matrix $\mathbf{C}(\xi, \mathrm{x})$, the solution of equation (9) is

$$
\begin{aligned}
\boldsymbol{Q}(x) & =\boldsymbol{C}(\xi, x)^{-1} \boldsymbol{D}(\xi, x) \boldsymbol{F}^{(x)}(\xi, x) \\
& =\boldsymbol{K}^{(x)}(\xi, x) \boldsymbol{F}^{(x)}(\xi, x)
\end{aligned}
$$

and $\mathbf{K}^{(x)}(\xi, \mathrm{x})=\mathbf{C}(\xi, \mathrm{x})^{-1} \mathbf{D}(\xi, \mathrm{x})$. Equation (10) can be written as

$$
\begin{gathered}
\boldsymbol{Q}(x)=\boldsymbol{K}(\xi, x) \boldsymbol{F}(\xi) \\
\boldsymbol{F}(\xi)=\left[f\left(\xi^{1}\right), . ., f\left(\xi^{g(1)}\right), ., f\left(\xi^{g(N(x))}\right), \ldots, f\left(\xi^{M}\right)\right]^{T}
\end{gathered}
$$

Where $M$ is the total number of nodes in the problem domain. Alternatively, one can write equation (11) as following

$$
Q_{I}(x)=\sum_{J=1}^{M} K_{I J} F_{J}, \quad I=1,2, \ldots, 8
$$

Where $F_{J}=f\left(\xi^{J}\right)$. The value of the function and its derivatives at the point $\mathbf{x}$ are defined in terms of values of the function at all nodes in the problem domain. Eight components of equation (13) for $1 \mathrm{D}$ case are given as following

$$
\begin{aligned}
& f(x)=Q_{1}(x)=\sum_{J=1}^{M} K_{1 J} F_{J} \\
& \frac{\partial f(x)}{\partial x_{1}}=Q_{2}(x)=\sum_{J=1}^{M} K_{2 J} F_{J} \\
& \frac{\partial^{2} f(x)}{\partial x_{1}^{2}}=2 ! Q_{3}(x)=\sum_{J=1}^{M} K_{3 J} F_{J} \\
& \frac{\partial^{3} f(x)}{\partial x_{1}^{3}}=3 ! Q_{4}(x)=\sum_{J=1}^{M} K_{4 J} F_{J} \\
& \frac{\partial^{4} f(x)}{\partial x_{1}^{4}}=4 ! Q_{5}(x)=\sum_{J=1}^{M} K_{5 J} F_{J} \\
& \frac{\partial^{5} f(x)}{\partial x_{1}^{5}}=5 ! Q_{6}(x)=\sum_{J=1}^{M} K_{6 J} F_{J} \\
& \frac{\partial^{6} f(x)}{\partial x_{1}^{6}}=6 ! Q_{7}(x)=\sum_{J=1}^{M} K_{7 J} F_{J} \\
& \frac{\partial^{7} f(x)}{\partial x_{1}^{7}}=7 ! Q_{8}(x)=\sum_{J=1}^{M} K_{8 J} F_{J}
\end{aligned}
$$

The formulation for $2 \mathrm{D}$ and $3 \mathrm{D}$ problems can be found [11-14].

\section{Modelling of the Semi-Trailer Chassis}

By using the 3D model of the semi-trailer chassis, 1D Euler Bernoulli beam model is presented in this section. As it is very well known and can be seen from the Figure 2, the semi-trailer chassis has a very complex structure. The deflection of the chassis regarding to the various loading conditions can be computed by using commercial finite element analysis software. But the aim of this study is not to compare the performance of meshless methods mentioned above with FEM software.

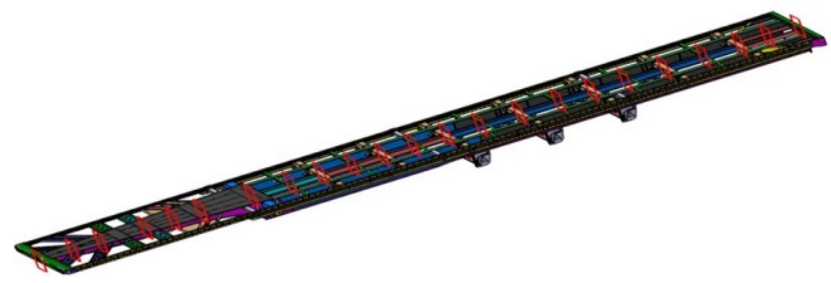

Fig. 2. 3D Model of a Semi Trailer Chassis

During the new product development process, the semitrailer chassis can be modified which are considered major modifications several times. For each major modification, to perform and repeat the finite element analysis with FEM software is a costly and time consuming activity because of re-meshing. Motivated by the fact that the performing and repeating finite element analysis is costly and time consuming, an alternative approach is investigated. 3D dimensional semi-trailer chassis is modelled as 1D dimensional beam based on Euler Bernoulli beam theory. To determine the moment of inertia of the beam is the most difficult part of mentioned modelling phase. It is found that the moment of inertia of the Euler Bernoulli beam can be defined as a function by using the moment of inertia values from the different sections of the chassis. It has to be mentioned that the selection of the sections is not a random activity; it is based on the design experience and engineering knowledge in terms of strength of materials. Totally 23 sections are selected to present the moment of inertia function of the semi-trailer chassis. The 23 sections can be seen from Figure 2. By using these moment of inertia values, the moment of inertia function of the 1D dimensional beam is obtained with POLYFIT function of MATLAB.

\section{Numerical Results}

The SSPH method is applied to solve the two problems of which are with different loading and boundary conditions in this section. The results of SSPH method employing different number of terms in the TSEs are compared with each other. Nonetheless, the SSPH method can be easily applied to any boundary value problem and complex domains in a systematic way.

\subsection{Simply Supported Beam with Partially Distributed Load}

A distributed load is applied to the simply supported beam shown in Figure 3 and according to this loading 
condition (case 1) the fourth order governing equation can be given as follows [20]

$$
\frac{d^{2}}{d x^{2}}\left(E I(x) \frac{d^{2} w}{d x^{2}}\right)=q(x)
$$

where the $\mathrm{E}$ is modulus of elasticity, $\mathrm{I}(\mathrm{x})$ is the moment of inertia of the cross section, $w$ is the deflection of the neutral axis and the $\mathrm{q}(\mathrm{x})$ is the distributed load.

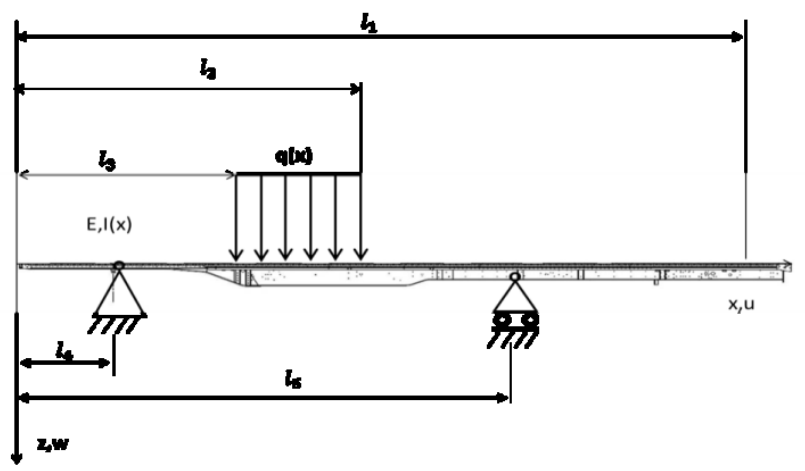

Fig. 3. Simply Supported Beam - Load Case 1

The physical parameters are as follows;

$l_{1}=13.6 \mathrm{~m}, l_{2}=5.95 \mathrm{~m}, l_{3}=4.45 \mathrm{~m}$,

$l_{4}=1.65 \mathrm{~m}, l_{5}=8.85 \mathrm{~m}$

Modulus of elasticity $\mathrm{E}$ is $210 \mathrm{GPa}$ and the distributed load q is set to $113513 \mathrm{~N} / \mathrm{m}$. The weight of the trailer chassis is neclegted. The boundary conditions are given as follows;

$$
\begin{gathered}
x=0, \quad \frac{d^{2} w}{d x^{2}}=0 \text { and } \frac{d I}{d x} \frac{d^{2} w}{d x^{2}}+I \frac{d^{3} w}{d x^{3}}=0 \\
x=l_{1}, \quad \frac{d^{2} w}{d x^{2}}=0 \text { and } \frac{d I}{d x} \frac{d^{2} w}{d x^{2}}+I \frac{d^{3} w}{d x^{3}}=0 \\
l_{2} \leq x \leq l_{3}, \frac{d^{2} w}{d x^{2}}=0, \frac{d^{2} I}{d x^{2}} \frac{d^{2} w}{d x^{2}}+2 E \frac{d I}{d x} \frac{d^{3} w}{d x^{3}}+E I \frac{d^{4} w}{d x^{4}} \\
=-q \\
x=l_{4}, \quad \frac{d^{2} w}{d x^{2}}=0 \text { and } w=-0.013 \mathrm{~m} \\
x=l_{5}, \quad \frac{d^{2} w}{d x^{2}}=0 \text { and } w=0 \mathrm{~m}
\end{gathered}
$$

The above forth order homogeneous variable coefficients boundary value problem is solved by using the SSPH method for the node distributions of 141, 273 and 545 in the problem domain employing different number of terms in the TSEs. The following Revised Super Gauss Function in [11] is used for each loading conditions as the weight function since it resulted in the least $\mathrm{L}_{2}$ error norms in numerical solutions presented in [11].

$$
W(x, \xi)=\frac{G}{(h \sqrt{\pi})^{\lambda}}\left\{\begin{array}{cc}
\left(36-d^{2}\right) e^{-d^{2}} & 0 \leq d \leq 6 \\
0 & d>6
\end{array}\right\}
$$

where $d=|x-\xi| / h$ is the radius of the support domain which is set to $6, h$ is the smoothing length, $\lambda$ is equal to the dimensionality of the space (i.e., $\lambda=1,2$ or 3 ) and $G$ is the normalization parameter having the values $1.04823,1.10081$ and 1.18516 for $\lambda=1,2$ and 3 , respectively. It is chosen that the smoothing length $h=1.5 \Delta$ for two adjacent nodes for the examples studied in this paper. Numerical results obtained by using the SSPH method employing different number of terms in the TSEs are compared with the experimental, and their convergence and accuracy features are evaluated by using the following global $\mathrm{L}_{2}$ error norm

$$
\| \text { Error } \|_{2}=\frac{\left[\sum_{j=1}^{m}\left(w_{\text {num }}^{j}-w_{\text {exact }}^{j}\right)^{2}\right]^{1 / 2}}{\left[\sum_{j=1}^{m}\left(w_{\text {exact }}^{j}\right)^{2}\right]^{1 / 2}} \times 100
$$

where $v_{\text {num }}^{j}$ is the value of numerical solution $v$ at the $j^{\text {th }}$ node and $v_{\text {exact }}^{j}$ is the value of analytical solution at the $j^{\text {th }}$ node.

Global $\mathrm{L}_{2}$ error norms of the solutions of SSPH method are given in Table 1, where numbers of nodes and terms in TSEs are varying. The results in Table 1 are obtained for the parameter values of $d$ and $h$ that yield the best accuracy. The compact support domain radius $d$ is equal to 6 and smoothing length $h=1.5 \Delta$. It is clear that, even with the same number of terms, solutions of the SSPH method agree very well with the analytical solution.

To evaluate the performance of the SSPH method, numerical solutions are obtained for 5 to 8 terms in the TSEs. It is observed that the rate of convergence of the numerical solution increases with an increase in the degree of complete polynomials. However, with the same number terms in the TSEs, the convergence rate of the SSPH method is decreasing even the number of nodes is increased in the problem domain.

Table 1. Global $\mathrm{L}_{2}$ error norm for different number of nodes and terms in the TSEs

\begin{tabular}{|c|c|c|c|}
\hline \multirow{2}{*}{$\begin{array}{c}\text { Number of } \\
\text { Terms }\end{array}$} & \multicolumn{4}{|c|}{ Number of Nodes in the Problem Domain } \\
\cline { 2 - 4 } & 141 & 273 & 545 \\
\hline 5 & 18.24 & 13.92 & 12.56 \\
\hline 6 & 17.58 & 12.89 & 11.46 \\
\hline 7 & 17.13 & 12.03 & 10.49 \\
\hline 8 & 16.76 & 11.07 & 9.63 \\
\hline
\end{tabular}

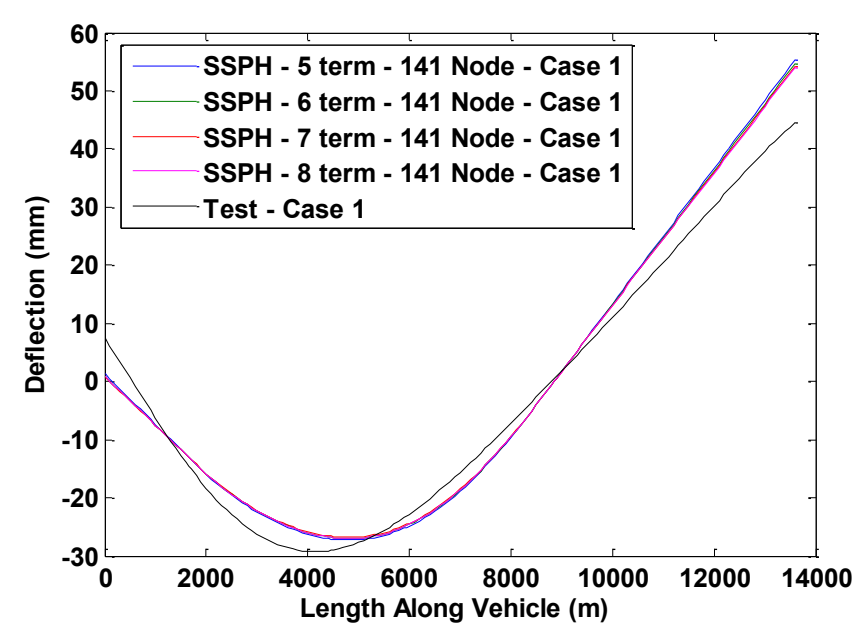

Fig. 4. Deflections along the $x$-axis computed by the SSPH method employing different number of terms in the TSEs and experimental results where 141 nodes are used 


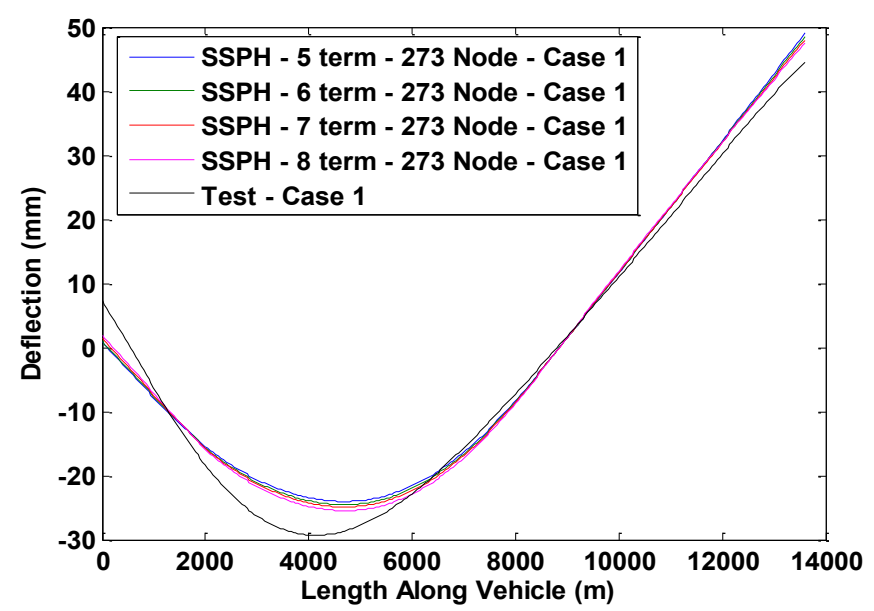

Fig. 5. Deflections along the $\mathrm{x}$-axis computed by the SSPH method employing different number of terms in the TSEs and experimental results where 273 nodes are used

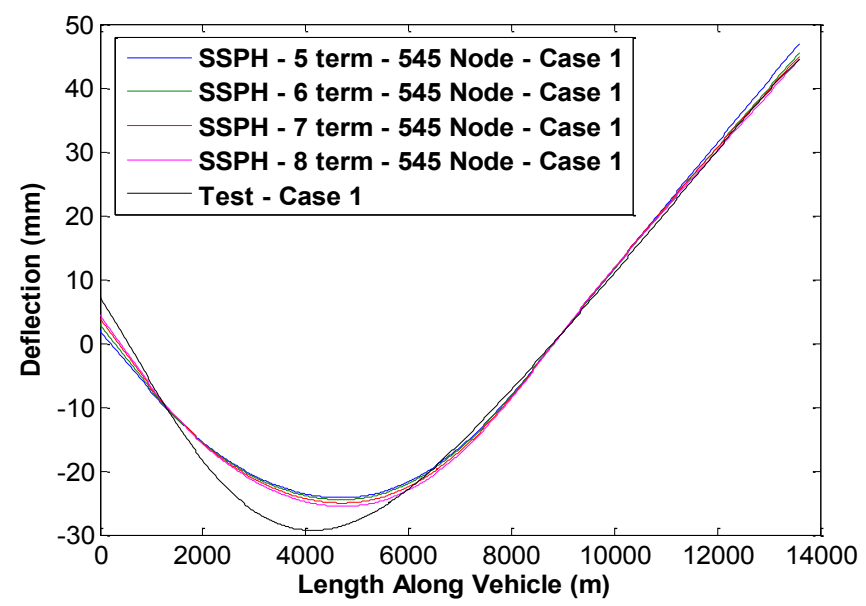

Fig. 6. Deflections along the $\mathrm{x}$-axis computed by the SSPH method employing different number of terms in the TSEs and experimental results where 545 nodes are used

It is observed in Figures 4 to 6 that accuracy of the SSPH method increases as the number of nodes and terms in the TSEs is increased.

\subsection{Simply Supported Indeterminate Beam with Partially Distributed Load}

Equally spaced distributed loads (case 2 - concrete blocks) are applied to the simply supported beam shown in Figure 7.

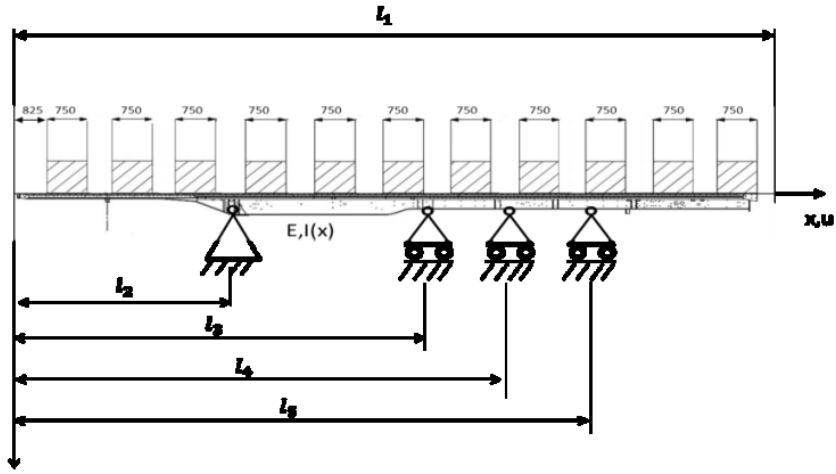

Fig. 7. Simply Supported Beam - Load Case 1
The physical parameters are as follows;

$l_{1}=13.6 \mathrm{~m}, l_{2}=3.75 \mathrm{~m}, l_{3}=7.5 \mathrm{~m}$,

$l_{4}=8.85 \mathrm{~m}, l_{5}=10.15 \mathrm{~m}$

Modulus of elasticity $\mathrm{E}$ is $210 \mathrm{GPa}$ and the an equally spaced distributed load q is set to $37575 \mathrm{~N} / \mathrm{m}$. The boundary conditions are given as follows;

$$
\begin{gathered}
x=0, \quad \frac{d^{2} w}{d x^{2}}=0 \text { and } \frac{d I}{d x} \frac{d^{2} w}{d x^{2}}+I \frac{d^{3} w}{d x^{3}}=0 \\
x=l_{1}, \quad \frac{d^{2} w}{d x^{2}}=0 \text { and } \frac{d I}{d x} \frac{d^{2} w}{d x^{2}}+I \frac{d^{3} w}{d x^{3}}=0 \\
x=l_{3}, \frac{d^{2} w}{d x^{2}}=0, w=0.0005 \mathrm{~m} \\
x=l_{4}, \quad \frac{d^{2} w}{d x^{2}}=0 \text { and } w=0.0014 \mathrm{~m} \\
x=l_{5}, \quad \frac{d^{2} w}{d x^{2}}=0 \text { and } w=0.0005 \mathrm{~m}
\end{gathered}
$$

Nodes at concrete blocks

$$
\frac{d^{2} I}{d x^{2}} \frac{d^{2} w}{d x^{2}}+2 E \frac{d I}{d x} \frac{d^{3} w}{d x^{3}}+E I \frac{d^{4} w}{d x^{4}}=-q
$$

Global $\mathrm{L}_{2}$ error norms of the solutions of SSPH method are given in Table 2, where numbers of nodes and terms in TSEs are varying. The results in Table 1 are obtained for the parameter values of $d$ and $h$ that yield the best accuracy. The compact support domain radius $d$ is equal to 6 and smoothing length $h=1.5 \Delta$. It is clear that, even with the same number of terms, solutions of the SSPH method agree very well with the analytical solution. To evaluate the performance of the SSPH method, numerical solutions are obtained for 5 to 8 terms in the TSEs. It is observed that the rate of convergence of the numerical solution increases with an increase in the degree of complete polynomials. Moreover, with the same number terms in the TSEs, the convergence rate of the SSPH method is increasing as the number of nodes is increased in the problem domain.

Table 2. Global $\mathrm{L}_{2}$ error norm for different number of nodes and terms in the TSEs

\begin{tabular}{|c|c|c|c|}
\hline \multirow{2}{*}{$\begin{array}{c}\text { Number of } \\
\text { Terms }\end{array}$} & \multicolumn{3}{|c|}{ Number of Nodes in the Problem Domain } \\
\cline { 2 - 4 } & 141 & 273 & 545 \\
\hline 5 & 35.89 & 33.95 & 27.42 \\
\hline 6 & 35.48 & 30.09 & 25.01 \\
\hline 7 & 33.75 & 28.69 & 22.61 \\
\hline 8 & 31.18 & 27.33 & 19.91 \\
\hline
\end{tabular}




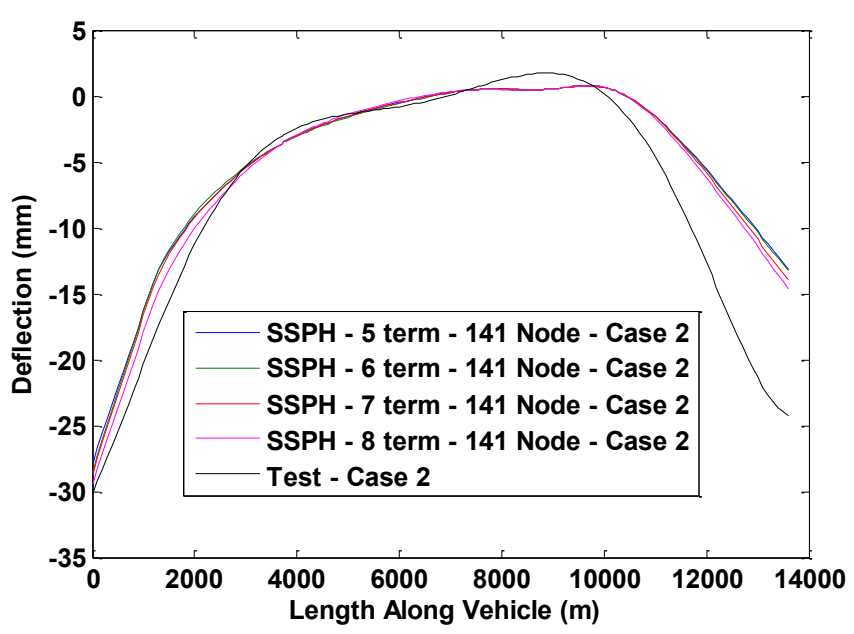

Fig. 8. Deflections along the $\mathrm{x}$-axis computed by the SSPH method employing different number of terms in the TSEs and experimental results where 141 nodes are used

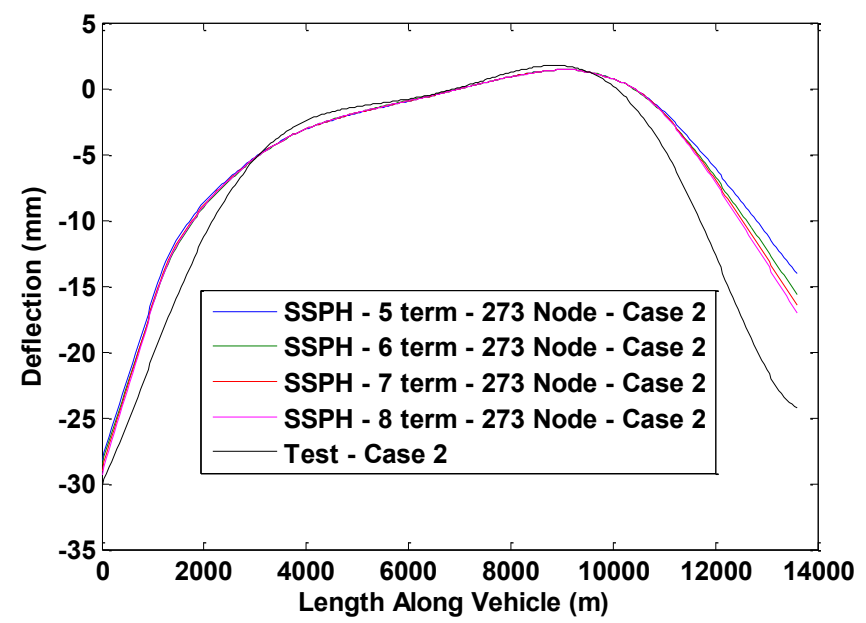

Fig. 9. Deflections along the $\mathrm{x}$-axis computed by the SSPH method employing different number of terms in the TSEs and experimental results where 273 nodes are used

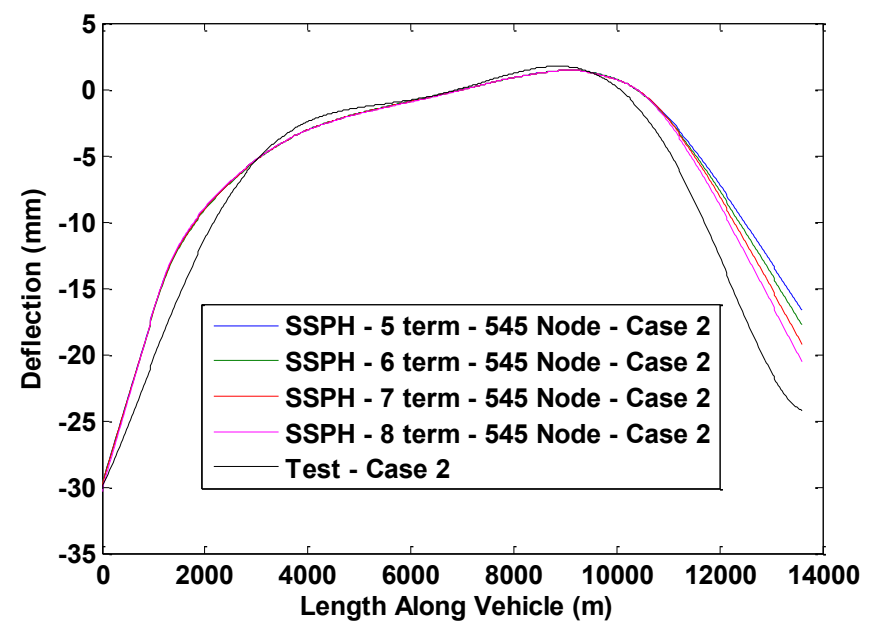

Fig. 10. Deflections along the $x$-axis computed by the SSPH method employing different number of terms in the TSEs and experimental results where 545 nodes are used
It is observed in Figures 8 to 10 that accuracy of the SSPH method increases as the number of nodes and terms in the TSEs is increased.

\section{Conclusion}

The deflections of a semi trailer chassis under various loading conditions are calculated based on 1D dimensional Euler Bernoulli beam. The 1D beam is modelled according to the CAD data and the moment of inertia function of the beam is defined by using polynomial function fitting. The numerical calculations are performed by using the SSPH method by employing different number of terms in the TSEs.

It is found that the simple 1D Euler Bernoulli beam modelled based on the 3D CAD data has enough details to obtain reasonable results by using numerical methods. So that during new product develeopment process it may be used to avoid the need of extra cost and time for repeating of FEM analysis. The SSPH method provides satisfactory results and convergence rate.

The first time the performance of the SSPH method for the fourth order non-homogeneous variable coefficents linear boundary value problems is evaluated. Moreover, it is found that the SSPH method is also useful for the solutions of the indeterminate beam problems. It is found that SSPH method yields more accurate results especially in the existence of eight terms in the TSEs. Classical Plate Theory and First Order Shear Deformation Theory which are not investigated in this paper will be the subject of future studies.

\section{Acknowledgements}

The author thanks to anonymous reviewers for their helpful suggestions.

\section{References}

[1] Lucy LB, A numerical approach to the testing of the fission hypothesis, Astron J 82, 1013-1024, 1977.

[2] Chen JK, Beraun JE, Jin CJ, An improvement for tensile instability in smoothed particle hydrodynamics, Comput Mech 23, 279-287, 1999.

[3] Chen JK, Beraun JE, Jin CJ, Completeness of corrective smoothed particlemethod for linear elastodynamics, Comput Mech 24, 273-285, 1999.

[4] Liu WK, Jun S, Zhang YF, Reproducing kernel particle methods, Int J Num Meth Fluids 20, 1081-1106, 1995.

[5] Liu WK, Jun S, Li S, Adee J, Belytschko T, Reproducing kernel particle methods for structural dynamics, Int J Num Meth Eng 38, 1655-1679, 1995.

[6] Chen JS, Pan C,Wu CT, Liu WK, Reproducing kernel particlemethods for large deformation analysis of nonlinear structures, Comput Method Appl Mech Eng 139, 195-227, 1996.

[7] Zhang GM, Batra RC, Modified smoothed particle hydrodynamics method and its application to transient problems, Comput Mech 34, 137-146, 2004. 
[8] Batra RC, Zhang GM, Analysis of adiabatic shear bands in elasto-thermo- viscoplastic materials by modified smoothed particle hydrodynamics (MSPH) method, J Comput Phys 201, 172-190, 2004.

[9] Zhang GM, Batra RC, Wave propagation in functionally graded materials by modified smoothed particle hydrodynamics (MSPH) method, J Comput Phys 222, 374-390, 2007.

[10] Batra RC, Zhang GM, Search algorithm, and simulation of elastodynamic crack propagation by modified smoothed particle hydrodynamics (MSPH) method, Comput Mech 40, 531-546, 2007.

[11] Zhang GM, Batra RC, Symmetric smoothed particle hydrodynamics (SSPH) method and its application to elastic problems, Comput Mech 43, 321-340, 2009.

[12] Batra RC, Zhang GM, SSPH basis functions for meshless methods, and comparison of solutions with strong and weak formulations, Comput Mech 41, 527$545,2008$.

[13] Tsai CL, Guan YL, Batra RC, Ohanehi DC, Dillard JG, Nicoli E, Dillard DA, Comparison of the performance of SSPH and MLS basis functions for two-dimensional linear elastostatics problems including quasistatic crack propagation, Comput Mech 51, 19-34, 2013.

[14] Karamanli A, Mugan A, Solutions of two-dimensional heat transfer problems by using symmetric smoothed particle hydrodynamics method, Journal of Applied and Computational Mathematics 1, 1-6, 2012.

[15] Karamanli A, Mugan A, Strong from meshless implementation of Taylor series method, Appl. Math. Comput. 219, 9069-9080,2013 .

[16] Karamanli A, Different Implementation Approaches of the Strong Form Meshless Implementation of Taylor Series Method, International Journal of Engineering Technologies, Vol. 1, No:3, 2015.

[17] Kaewumpai, S., Luadsong, A., Two-field-variable meshless method based on moving kriging interpolation for solving simply supported thin plates under various loads. J. King Saud Univ. Sci., 1018-3647, 2014.

[18] Yimnak, K., Luadsong, A., A local integral equation formulation based on moving kriging interpolation for solving coupled nonlinear reaction-diffusion equations. Adv. Math. Phys., 2014.

[19] Zhuang, X., Zhu, H., Augarde, C., The meshless Shepard and least squares (MSLS) method, Comput Mech, 53, 343-357, 2014.

[20] Wang JM, Reddy JN, Shear deformable beams and plates, relationships with classical solutions. Elsevier, Oxford, 2000. 